\title{
BMJ Open Clinical, neurocognitive and demographic factors associated with functional impairment in the Australian Brain and Mind Youth Cohort Study (2008-2016)
}

\author{
Rico S C Lee, ${ }^{1,2}$ Daniel F Hermens, ${ }^{1}$ Sharon L Naismith, ${ }^{1,3}$ Manreena Kaur, ${ }^{1,4}$ \\ Adam J Guastella, ${ }^{1}$ Nick Glozier, ${ }^{1,5}$ Jan Scott, ${ }^{6,7}$ Elizabeth M Scott, ${ }^{1}$ Ian B Hickie ${ }^{1}$
}

To cite: Lee RSC, Hermens DF, Naismith SL, et al. Clinical, neurocognitive and demographic factors associated with functional impairment in the Australian Brain and Mind Youth Cohort Study (2008-2016). BMJ Open 2018;8:e022659. doi:10.1136/ bmjopen-2018-022659

- Prepublication history for this paper is available online. To view these files please visit the journal online (http://dx.doi org/10.1136/bmjopen-2018022659).

Received 1 March 2018 Revised 17 0ctober 2018 Accepted 2 November 2018

Check for updates

(C) Author(s) (or their employer(s)) 2018. Re-use permitted under CC BY-NC. No commercial re-use. See rights and permissions. Published by BMJ.

For numbered affiliations see end of article.

Correspondence to

Dr Rico S C Lee;

rico.lee@monash.edu

\section{ABSTRACT}

Objectives We sought to determine the unique and shared contributions of clinical, neurocognitive and demographic factors to functional impairment in a large, transdiagnostic, clinical cohort of adolescents and young adults.

Design Cross-sectional baseline data from a prospective, cohort study.

Setting Help-seeking youth referred from outpatient services were recruited to the Brain and Mind Youth Cohort (2008-2016) in Sydney, Australia.

Participants In total, 1003 outpatients were recruited, aged between 12 and 36 years (mean $=20.4$ years, $54 \%$ female), with baseline diagnoses of affective, psychotic, developmental or behavioural disorders.

Interventions Treatment as usual.

Primary outcome measures Social and occupational functioning was used to index level of functional impairment. Structural equation modelling was used to examine associations between neurocognition, core clinical symptoms and alcohol and substance use, and clinician-rated and researcher-rated functional impairment. Moderator analyses were conducted to determine the potential influence of demographic and clinical factors (eg, medication exposure).

Results Independent of diagnosis, we found that neurocognitive impairments, and depressive, anxiety and negative symptoms, were significantly associated with functioning. The association of neurocognition with social and occupational functioning remained significant even when constraining for age (15-25-year-olds only) or diagnosis (affective disorders only) in the final model. Conclusions This study demonstrated that, in a clinically representative sample of youth, the key determinants of functioning may not be disorder specific. Further, evidence of neurocognitive dysfunction suggests that interventions that target cognition and functioning should not necessarily be reserved just for older adults with established illness.

\section{INTRODUCTION}

In recent decades, early intervention services for youth with emerging mental disorders

\section{Strengths and limitations of this study}

- This was one of the largest studies to date $(n>1000)$ to examine the associations between a broad range of illness characteristics and functional impairment in a mostly adolescent and young adult, clinical sample.

- Given the transdiagnostic approach, this study was equipped to disentangle the shared and unique associations between core illness phenotypes and functional impairment across a range of common mental disorders.

- The use of latent-variable, structural equation modelling controlled for aspects of measurement imprecision.

have extended their targets beyond those at risk of psychosis to also encompass those presenting with mood as well as other developmental and anxiety disorders. This approach creates several significant challenges. For example, some youth with depressive and anxiety disorders will ultimately develop psychotic or bipolar disorders; likewise, only a proportion of those receiving a diagnosis of bipolar disorders will consistently receive this diagnosis over the following 10 years. ${ }^{1}$ The lack of diagnostic stability in help-seeking youth reflects the evolving disease process and means that the illness trajectory is less certain than for older adults with established illness. ${ }^{2}{ }^{3}$ From a research perspective, the use of dimensional approaches to phenomenology has helped us to understand illness progression in these early clinical stages, while from a clinical perspective, care and treatment have increasingly considered transdiagnostic interventions addressing core factors that may influence prognosis irrespective of cross-sectional diagnosis (eg, 
anxiety, depressive or negative symptoms; sleep disturbances). ${ }^{45}$ These approaches have highlighted that, in youth, a more meaningful measure of outcome may be functioning rather than change in diagnosis-specific symptoms. There is evidence to support this approach as level of functioning or disengagement (eg, not being in education, employment or training, referred to as being NEET) is associated with early transition to major mental disorder ${ }^{6}$ and with poor outcome of acute illness episodes. $^{78}$ However, to optimise interventions that target functioning it is important to understand the factors that contribute to level of functioning at clinical presentation. For instance, in addition to transdiagnostic symptom dimensions, it is likely that other factors such as neurocognitive functioning and alcohol or substance use will also affect overall functioning. Disentangling the contribution and magnitude of any effects of these factors on functioning is important to determine which factors may be amenable to modification and allow clinicians to design a multidimensional intervention package.

The proposal that social and occupational functioning should be a primary target for mental health interventions is not new and is increasingly promoted for older adults with established illness. For example, senior policy experts in the USA have stipulated that recovery-oriented treatments should form the overarching goal of mental healthcare and the foundation of strategic health policy. ${ }^{9}$ The recognition that more personalised interventions are urgently needed to enhance functioning and quality of life rather than simply targeting diagnosis-specific symptoms in a one-size-fits-all approach is also emphasised by the WHO. ${ }^{10}$ Given this interest in enhancement of functioning across all stages of mental illness and for youth and adults presenting to mental health services, it is therefore useful to examine the role of other key clinical (eg, medication exposure) and demographic factors (eg, age, gender) in determining functioning which would contribute to prognosis and attempts at personalised medicine.

Most path modelling studies to date have used small, single-diagnosis or dual-diagnosis cohorts, predominantly in individuals with a chronic mental illness. Findings consistently demonstrate that neurocognition and negative symptoms are robust predictors of functional outcome in schizophrenia and bipolar disorder. ${ }^{7112}$ By contrast, the impact of affective and positive symptoms on functioning remains more equivocal. More recently, these findings have been replicated in large cohort studies, ${ }^{13} 14$ although the vast majority of existing studies have focused exclusively on schizophrenia. There have been no well-powered studies examining a mental disorder other than schizophrenia, such as affective disorders, despite depression being the leading cause of disability worldwide. ${ }^{15}$ Studies have also largely sidestepped the issue of psychotropic medication use. Furthermore, given that more than $75 \%$ of mental illnesses emerge before the age of $25,{ }^{3}$ examining younger cohorts is critically important for the development of novel approaches to early intervention since most studies to date have targeted older individuals. ${ }^{15}$

In order to build on prior research, a transdiagnostic and dimensional approach is ideally positioned to disentangle the factors associated with functioning. Key to this research strategy is the examination of shared constructs (eg, neurocognition) with clear links to pathophysiology $^{16-18}$ which can inform novel therapeutics that target specific neural circuitries. ${ }^{17} 19$ Transdiagnostic studies are also able to harness the variance across disorders, with the goal of developing robust, unifying models that are explanatory in nature. ${ }^{2}$ Data showing that physiological and genetic risk factors for mental illness extend across, rather than are bound by, traditional diagnoses,${ }^{20}$ further supports this paradigm, as does the frequent prescription of psychotropic medications for off-label use across diagnostic boundaries. ${ }^{21}$ Transdiagnostic studies are also superior to single-diagnosis case-control studies in that they can determine which relationships are shared across various diagnoses and which are unique to a particular disorder.

In this study, we sought to determine whether (1) neurocognition, (2) core clinical dimensions and (3) alcohol and substance use are associated with social and occupational functioning and the magnitude of these associations. The rationale for examining clinical symptoms and functioning alongside neurocognition, sleep changes and substance use is underscored by a recent systematic review highlighting the transdiagnostic relevance of these key domains in youth with mental illness. ${ }^{22}$ In keeping with prior research, ${ }^{712} 23$ it was hypothesised that neurocognition and negative symptoms would make the greatest contribution to level of social and occupational functioning irrespective of the cross-sectional diagnosis applied to cases at the time of inclusion in the cohort. Given the high degree of heterogeneity expected in a transdiagnostic youth sample, we secondarily sought to determine the influence of demographic (eg, age, gender) and clinical factors (eg, diagnosis, medication exposure) on findings.

\section{METHODS}

Data included in the current study represent the baseline assessments conducted at entry to the cohort study and were collected between April 2008 and May 2016.

\section{Participants}

Participants were recruited into the Brain and Mind Youth Cohort from youth mental health outpatient services at the Brain and Mind Centre. ${ }^{24}{ }^{25}$ Referred participants were 12-36years of age and presented with a major affective, psychotic or developmental/behavioural syndrome. Participants were excluded if they (or their guardians, if aged under 16years) were unwilling or unable to provide written informed consent, or if they had a pre-existing neurological condition, clinically assessed impaired English language skills and/or intellectual disability 
Table 1 Demographic, clinical and functional characteristics across diagnostic subgroups

\begin{tabular}{|c|c|c|c|c|c|c|c|c|c|c|}
\hline & \multicolumn{2}{|c|}{$\begin{array}{l}\text { Depression* } \\
(\mathrm{n}=449)\end{array}$} & \multicolumn{2}{|c|}{$\begin{array}{l}\text { Bipolart } \\
(n=178)\end{array}$} & \multicolumn{2}{|c|}{$\begin{array}{l}\text { Psychosis } ¥ \\
(n=193)\end{array}$} & \multicolumn{2}{|c|}{$\begin{array}{l}\text { Anxiety§ } \\
(n=109)\end{array}$} & \multicolumn{2}{|c|}{$\begin{array}{l}\text { Dev/behav } \\
(n=74)\end{array}$} \\
\hline & $\mathbf{M}$ & SD & M & SD & M & SD & M & SD & M & SD \\
\hline Education (years) & 11.6 & 2.4 & 12.3 & 2.2 & 12.0 & 2.4 & 11.5 & 2.7 & 10.0 & 2.8 \\
\hline BPRS depression (/7) & 2.4 & 0.8 & 2.2 & 0.8 & 2.1 & 0.9 & 2.3 & 0.9 & 1.7 & 0.7 \\
\hline BPRS negative (/7) & 1.5 & 0.6 & 1.3 & 0.5 & 1.9 & 0.8 & 1.5 & 0.7 & 1.5 & 0.6 \\
\hline BRPS disorientation (/7) & 1.2 & 0.5 & 1.1 & 0.5 & 1.2 & 0.6 & 1.2 & 0.5 & 1.2 & 0.5 \\
\hline HDRS sleep (/6) & 2.0 & 1.8 & 1.7 & 1.7 & 1.3 & 1.6 & 1.5 & 1.5 & 1.8 & 1.7 \\
\hline AUDIT alcohol use (/40) & 6.8 & 7.4 & 9.0 & 8.4 & 6.2 & 8.0 & 4.4 & 6.4 & 5.1 & 7.5 \\
\hline \multirow[t]{2}{*}{ SOFAS } & 61.8 & 10.7 & 63.9 & 11.5 & 55.9 & 12.1 & 63.2 & 11.1 & 61.7 & 9.8 \\
\hline & $\mathbf{N}$ & $\%$ & $\mathbf{N}$ & $\%$ & $\mathbf{N}$ & $\%$ & $\mathbf{N}$ & $\%$ & $\mathbf{N}$ & $\%$ \\
\hline Gender (female) & 277 & 61.7 & 129 & 72.5 & 58 & 30.1 & 58 & 53.2 & 20 & 27.0 \\
\hline Medicated $^{\star \star}$ & 231 & 60.0 & 128 & 77.6 & 140 & 77.3 & 37 & 45.7 & 32 & 49.2 \\
\hline Antidepressants & 201 & 52.2 & 63 & 38.2 & 55 & 30.4 & 25 & 30.9 & 12 & 18.5 \\
\hline Lithium/anticonvulsants & 28 & 7.3 & 69 & 41.8 & 24 & 13.3 & 6 & 7.4 & 3 & 4.6 \\
\hline Antipsychotics & 71 & 18.4 & 77 & 46.7 & 125 & 69.1 & 9 & 11.1 & 8 & 12.3 \\
\hline
\end{tabular}

*Major depressive disorder ( $n=313)$, dysthymic disorder $(n=4)$, depressive disorder not otherwise specified $(n=132)$.

†Bipolar I disorder $(n=13)$, bipolar II disorder $(n=25)$, cyclothymic disorder $(n=1)$, bipolar disorder not otherwise specified $(n=139)$.

$\ddagger$ Schizophrenia $(n=53)$, schizophreniform disorder $(n=15)$, schizoaffective disorder $(n=26)$, brief psychotic disorder $(n=11)$, substance-induced psychotic disorder $(n=14)$, psychotic disorder not otherwise specified $(n=74)$.

§Panic disorder $(n=4)$, social phobia $(n=29)$, obsessive-compulsive disorder $(n=11)$, post-traumatic stress disorder $(n=5)$, generalised anxiety disorder $(n=60)$.

ПAsperger's disorder $(n=16)$, attention-deficit/hyperactivity disorder $(n=47)$, conduct disorder $(n=7)$, oppositional defiant disorder ( $n=4)$. ${ }^{* *}$ Medication data were available in 877 individuals (87.4\%), with missing data for the typologies of depression $(n=64)$, bipolar $(n=13)$, psychosis $(n=12)$, anxiety $(n=28)$, developmental $(n=9)$.

AUDIT, Alcohol Use Disorders Identification Test; BPRS, Brief Psychiatric Rating Scale; dev/behav, developmental/behavioural; HDRS, Hamilton Depression Rating Scale; SOFAS, Social and Occupational Functioning Assessment Scale; WHO-ASSIST, WHO-Alcohol, Smoking and Substance Involvement Screening Test.

that precluded completion of study self-ratings. Eligible participants completed a series of observer and self-rated questionnaires.

\section{Procedure}

Treating clinicians recorded clinical diagnoses, and these were reviewed at consensus meetings by senior, treating psychiatrists (eg, IBH, EMS) and formal diagnoses recorded based on the Diagnostic and Statistical Manual of Mental Disorders - Fourth Edition (Text Revision) (DSM-IV-TR); table 1 provides details of diagnoses and sample characteristics). Any disagreements in diagnosis were resolved at these consensus meetings with the relevant treating team. All participants received their prescribed course of medications and interventions, as independently determined in consultation with their treating clinicians.

Board-certified treating clinicians (ie, consultant psychiatrists, clinical psychologists, mental health nurses) provided an evaluation of each participant's social and occupational functioning. Next, board-certified clinical psychologists, clinical neuropsychologists or trained research psychologists (ie, graduate-level academic psychologists, supervised by RSCL to ensure a sufficient level of inter-rater reliability) conducted structured clinical interviews, neuropsychological testing, as well as an additional assessment of social and occupational functioning to improve the reliability of this single, clinician-rated score (approximately $80 \%$ were conducted within a month of the treating clinician assessment). 


\section{Measures}

- Social and occupational functioning was indexed using the Social and Occupational Assessment Functioning Scale (SOFAS). ${ }^{26}$ Scores were averaged across the treating clinician and researcher assessments (intraclass correlation coefficient $=0.70$ ), as previously done. $^{27}$ This composite score was derived to obtain a more reliable estimate of real-world functioning and, secondarily, to conserve free parameters and increase stability of parameter estimates. ${ }^{23}$ A higher score denotes better functioning.

- Neurocognition was assessed using a broad neuropsychological battery with demographic normative-adjustments (previously described ${ }^{23}$ ) and was chosen on the basis of sound psychometric properties ${ }^{29}$ and relevance to the disorders under study. ${ }^{30}$ Predicted IQ was estimated using the Wechsler Test of Adult Reading or Wide Range Achievement Test-fourth edition (for participants younger than 16 years). Psychomotor speed and mental flexibility were measured using Trail Making Test-Part A (TMT-A) and Part B (TMTB). Verbal learning and memory were indexed using the five-trial total and delayed recall scores from the Rey Auditory Verbal Learning Test. Verbal fluency was composed of the letter (F-A-S) and category (animals) fluency subtests of the Controlled Oral Word Association Test. A higher score indicates better functioning.

- Core clinical symptom dimensions were measured across two validated scales. Symptoms were rated on the expanded Brief Psychiatric Rating Scale (BPRS) using empirically derived symptom subscores (depression and anxiety, mania, positive symptoms, negative symptoms and disorientation ${ }^{31}$ ). The BPRS does not capture sleep profiles as a separate dimension so, as in previous studies, ${ }^{32}$ disturbed sleep was indexed using the sum of the three sleep items from the Hamilton Depression Rating Scale. ${ }^{33}$ A higher score denotes greater severity of symptoms.

- Alcohol and substance use were measured across two validated scales. Alcohol use was indexed using the Alcohol Use Disorders Identification Test ${ }^{34}$ total score. Substance use for tobacco, cannabis and other illicit substances was measured using the 'current frequency' subscale (past 3 months) from the WHOAlcohol, Smoking and Substance Involvement Screening Test questionnaire. ${ }^{35}$ A higher score indicates greater alcohol or substance use.

\section{Patient and public involvement}

Participants were not involved in the development of research question(s), design and outcome measures, nor was the study informed by their priorities, experience and preferences. We did not formally assess the burden of time required to participate in the research.

\section{Data analysis}

Statistical analyses were conducted using IBM SPSS V.20.0 and AMOS V.20.0. Maximum likelihood estimation
(MLE) was employed for all structural equation modelling (SEM) analyses. MLE was chosen as it is the most robust approach in the potential event of statistical assumption violations and performs best in heterogeneous samples. ${ }^{36}$ Missing data were also handled by MLE which does not involve data imputation, but uses all available data to compute maximum likelihood estimates. Diagnostic and demographic data were available for all participants. In total, $9.1 \%$ of data were missing for functioning, $18.8 \%$ for neurocognition, $12.7 \%$ for clinical symptoms and disturbed sleep, and $17.7 \%$ were missing for alcohol and substance use. Each analysis had $>80 \%$ of cases with complete data. Additional analyses revealed that data were not missing at random, and missing data were more likely to occur in younger participants (Welch's $\mathrm{F}(1102.54)=4.85, \mathrm{p}<0.05$ ) and in those with an anxiety disorder $\left(\chi^{2}(4)=26.09, \mathrm{p}<0.001\right)$, although the effect sizes were small (Cohen's $\mathrm{d}=0.26$ and Cramer's $\mathrm{V}=0.16$, respectively).

Normality was assessed through inspection of Q-Q plots, given inferential measures of non-normality (eg, Shapiro-Wilk statistic) are overly sensitive in large datasets and almost always return a significant finding. ${ }^{37}$ All endogenous variables (eg, SOFAS) met normality assumptions on visual inspection. Based on visual inspection of the frequency histograms and assessment of the Q-Q plot, the predictor/exogenous variables that departed from normality were positive symptoms, negative symptoms, mania, disorientation, TMT-A and TMT-B which were all observed to have a slight positive skew (no others were skewed). Prior studies have found that MLE methods are robust in cases where variables depart from normality where $n>600$, as in the present case. ${ }^{38}$ However, other approaches to non-normal data, such as asymptotically distribution free SEM, require no missing data and would unsatisfactorily affect the generalisability of findings as well as statistical power in the current analyses. As such, we used the MLE approach.

We first used SEM to evaluate the best-fitting measurement model for the following predictors: (1) neurocognition, (2) clinicalsymptoms and disturbed sleep, and (3) alcoholand substance use. Then, we used SEM to test the structural model (ie, the relationship between predictors and social and occupational functioning) at both the single-predictor and the overall levels in order to explore potential predictors and delineate unique contributions. This was done in a two-step process-first, by testing individual predictors and then by testing the combined predictors - to quantify the amount of overlapping and unique explanatory power. All analyses used a model-trimming approach through an iterative process in which non-significant paths with the smallest contribution were sequentially eliminated from a saturated model (where all variables were allowed to freely co-vary), until a best fitting model was derived to best explain the relationships between predictors and functional impairment. Finally, modification indices generated by AMOS were used to optimise model fit (ie, to inform which 
paths and parameters should be added or removed to increase model adequacy), although these were only used when deemed theoretically meaningful. Residuals were allowed to correlate if theoretically justified (eg, common measurement variance between neuropsychological subtests).

Model fit was determined using: (1) the absolute fit $\chi^{2}$ statistic and (2) the relative fit indices: Bentler Comparative Fit Index (CFI ${ }^{39}$ Bentler-Bonnett Non-normed Fit Index $\left(\mathrm{NFI}^{40}\right.$ and Root Mean Square Error of Approximation (RMSEA ${ }^{41}$ with $90 \%$ CI. An excellent-fitting model is typically indicated by a non-significant $\chi^{2}$ test (indicating a non-significant difference between the covariance matrix of the data and the model), a CFI and NFI of greater than 0.90 (indicating that the current model was superior to a null model where all paths are constrained to zero), and a RMSEA of less than 0.05 with an upper CI bound of less than 0.08 (indicating that the error of approximation of the model compared with the data was acceptable). In small samples (ie, less than 200), the $\chi^{2}$ statistic has been shown to be an adequate index of absolute model fit. ${ }^{36}$ However, as sample size increases, the $\chi^{2}$ statistic (relative to a constant $\mathrm{df}$ ) disproportionately increases, and is nearly always significant and inappropriately rejects the model irrespective of specified parameters. ${ }^{42}{ }^{43}$ An alternative solution is to compute a relative $\chi^{2} / \mathrm{df}$ ratio, with a value between 2 and 5 considered excellent to adequate fit, ${ }^{424-46}$ although primary emphasis will be placed on the relative fit indices as is the established convention. ${ }^{13}$

Moderator analyses were conducted allowing a model to be tested in separate subgroups, comparing the parameter estimates to determine how predictors of social and occupational functioning in the final model are moderated by demographic and clinical factors (these were dichotomous to maintain statistical power within subgroups for this categorical procedure). For instance, the median-split on age was performed to determine whether the model held for both younger and older individuals while maintaining statistical power. We sought to specifically test whether predictors in affective-spectrum disorders (anxiety, depressive and bipolar disorders) were similarly associated with functional impairment compared with psychotic, developmental or behavioural conditions. We chose to include primary affective disorders (ie, major depression, bipolar disorder or an anxiety disorder) as a moderator since these disorders have been shown to carry less neurocognitive burden in recentonset mood disorders ${ }^{47} 48$ and, as such, could potentially influence the role of neurocognition and the magnitude of effects in the statistical models.

\section{RESULTS}

\section{Sample characteristics}

In total, 1003 patients were recruited. As shown in tables 1 and 2, cross-sectional diagnoses were composed of depressive $(n=449)$, bipolar $(n=178)$, psychotic $(n=193)$, anxiety $(\mathrm{n}=109)$, and developmental or behavioural disorders $(\mathrm{n}=74)$. The mean age was 20.4 years $(\mathrm{SD}=4.7)$, with $54.0 \%$ being female $(\mathrm{n}=542)$. Mean educational attainment was 11.7 years $(\mathrm{SD}=2.5)$, with an average predicted IQ of 101.9 ( $\mathrm{SD}=10.8)$. The mean SOFAS score was 61.2 ( $\mathrm{SD}=11.4)$, indicating moderate levels of impairment. Of the participants with medication data available $(87.4 \%$, $\mathrm{n}=877), 64.8 \%$ were prescribed psychotropic medications $(n=568)$. Of these 568 cases, $40.6 \%$ were prescribed an antidepressant $(n=356), 14.8 \%$ were prescribed lithium or an anticonvulsant $(\mathrm{n}=130), 33.1 \%$ were prescribed any antipsychotic $(\mathrm{n}=290)$ and $4.8 \%$ were prescribed a stimulant $(n=42)$.

\section{Single-predictor models}

A. Neurocognition. Inspecting the screen plot, exploratory factor analyses identified two potential latent structures. The one-factor model was a very good fit for the data $\left(\chi^{2}=57.3, \mathrm{df}=17, \mathrm{p}<0.001, \mathrm{CFI}=0.980, \mathrm{NFI}=0.972\right.$, RMSEA $=0.055,90 \%$ CI 0.040 to 0.071 ), and was a better fit than a two-factor model, whereby trails A, trails $B$ and IQ loaded on one latent factor, and IQ, Rey total, Rey delay, FAS and animals loaded on a second

Table 2 Neuropsychological functioning across diagnostic subgroups

\begin{tabular}{|c|c|c|c|c|c|c|c|c|c|c|}
\hline & \multicolumn{2}{|c|}{$\begin{array}{l}\text { Depression } \\
(\mathrm{n}=449)\end{array}$} & \multicolumn{2}{|c|}{$\begin{array}{l}\text { Bipolar } \\
(n=178)\end{array}$} & \multicolumn{2}{|c|}{$\begin{array}{l}\text { Psychosis } \\
(n=193)\end{array}$} & \multicolumn{2}{|l|}{$\begin{array}{l}\text { Anxiety } \\
(n=109)\end{array}$} & \multicolumn{2}{|c|}{$\begin{array}{l}\text { Dev/behav } \\
(n=74)\end{array}$} \\
\hline & M & SD & M & SD & $\mathbf{M}$ & SD & M & SD & $\mathbf{M}$ & SD \\
\hline Trails A† & -0.01 & 1.32 & 0.16 & 1.06 & -0.31 & 1.01 & 0.12 & 0.96 & -0.03 & 1.07 \\
\hline Trails B† & -0.44 & 1.53 & -0.47 & 1.92 & -1.22 & 2.23 & -0.50 & 1.59 & -0.85 & 2.16 \\
\hline FAS† & -0.31 & 1.15 & -0.04 & 1.07 & -0.56 & 1.00 & -0.37 & 1.15 & -0.86 & 1.09 \\
\hline Animals $†$ & 0.23 & 1.20 & 0.45 & 1.25 & -0.28 & 1.08 & 0.26 & 1.17 & -0.03 & 0.94 \\
\hline
\end{tabular}

*Age-adjusted; normative $M=100 ; S D=15$.

†Demographically adjusted; normative $\mathrm{M}=0.00 ; \mathrm{SD}=1.00$.

Dev/behav, developmental/behavioural. 

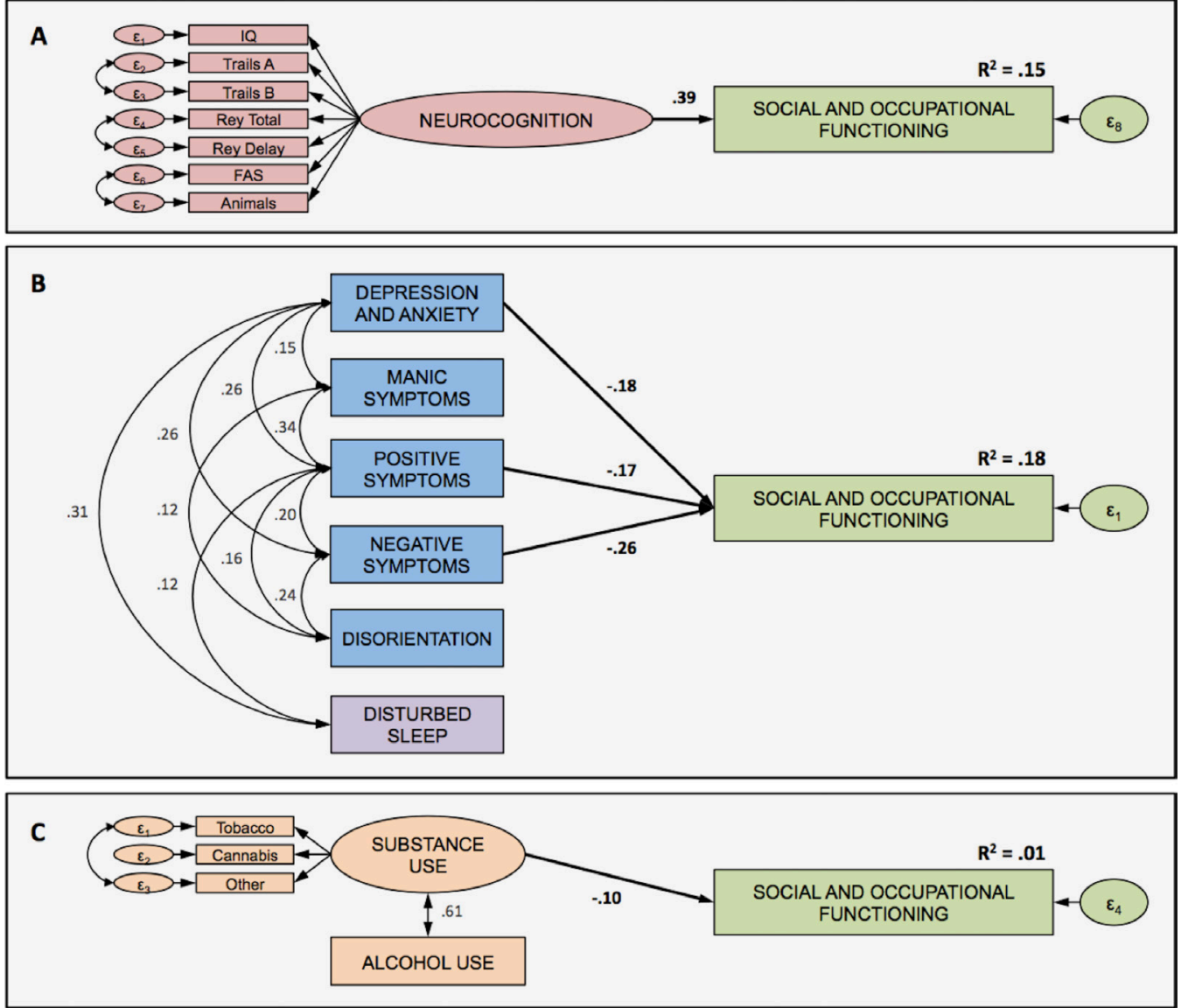

Figure 1 Combined measurement and structural models for functioning and (A) neurocognition, (B) core clinical symptoms and $(C)$ alcohol and substance use. All unidirectional (correlation) and directional (regression) paths are significant at $p<0.001$ (except path between substance use and functional outcome; where $p<0.05$ ) Factor loadings for $(A)$ neurocognition (all $p$ values $<0.001)$ : IQ (0.58), trails A ( -0.51$)$, trails B (-0.55), Rey total $(0.69)$, Rey delay $(0.59)$, FAS $(0.57)$, animals $(0.51)$. Factor loadings for $(C)$ substance use (all p values $<0.001)$ : tobacco $(0.81)$, cannabis $(0.64)$, other $(0.68)$.

latent factor $\left(\chi^{2}=76.499, \mathrm{df}=11, \mathrm{p}<0.001, \mathrm{CFI}=0.967\right.$, $\mathrm{NFI}=0.962$, RMSEA $=0.077,90 \%$ CI 0.061 to 0.094$)$. Factor loadings on the one-factor model were all significant and ranged from 0.51 to 0.69 (see figure 1A). Neurocognition was a significant contributor to functional level $(\beta=0.39, p<0.001)$, explaining $15 \%$ of the variance.

B. Core clinical symptom dimensions. Only three clinical dimensions (depression and anxiety $(\beta=-0.18, p<0.001)$, positive symptoms $(\beta=-0.17, \mathrm{p}<0.001)$ and negative symptoms $(\beta=-0.26, p<0.001))$ were associated with functioning, whereas mania and disorientation were not significantly associated ( $p$ values $>0.05$ ). The model demonstrated excellent fit $\left(\chi^{2}=8.6, \mathrm{df}=8, \mathrm{p}<0.379\right.$, $\mathrm{CFI}=0.999, \mathrm{NFI}=0.986$, RMSEA $=0.009,90 \%$ CI 0.000 to 0.043 ), with the three dimensions explaining a total of $18 \%$ of the variance in functioning (see figure 1B).

C. Alcohol and substance use. Exploratory factor analyses determined that alcohol use did not load with the other substance use variables. Only a two-factor latent model was possible given the number of observed variables and statistical constraints. The two-factor model emerged as an excellent fit for the data $\left(\chi^{2}=7.4\right.$, $\mathrm{df}=4, \mathrm{p}<0.116, \mathrm{CFI}=0.995, \mathrm{NFI}=0.990, \mathrm{RMSEA}=0.033$,
$90 \%$ CI 0.000 to 0.069 ), whereby tobacco, cannabis and other illicit substance use loaded on a single 'substance use' latent variable as distinct from alcohol use (figure 1C). Only substance use was predictive of functioning $(\beta=-0.10, p<0.05)$, explaining $1 \%$ of the variance.

\section{Final model}

In the overall model, all the factors identified in the single predictor models remained significant, except for substance use (figure 2). Neurocognition showed the strongest unique contribution to social and occupational functioning $(\beta=0.36$, $\mathrm{p}<0.001)$; depressive symptoms were next $(\beta=-0.24, \mathrm{p}<0.001)$, followed by negative symptoms $(\beta=-0.15, \mathrm{p}<0.001)$ and finally positive symptoms $(\beta=-0.10, p<0.001)$. Together, these four clinical features independently accounted for $31 \%$ of the variance in functioning, with the final model being a very good fit for the data $\left(\chi^{2}=279.8, \mathrm{df}=119, \mathrm{p}<0.000, \mathrm{CFI}=0.956\right.$, $\mathrm{NFI}=0.926$, RMSEA $=0.037,90 \%$ CI 0.031 to 0.042$)$. Mania, disorientation and alcohol and substance use all significantly correlated with these four significant features ( $p$ values $<0.05$ ).

\section{Moderator analyses}

- Age. As shown in table 3, positive symptoms were no longer a significant contributor to functioning in the 


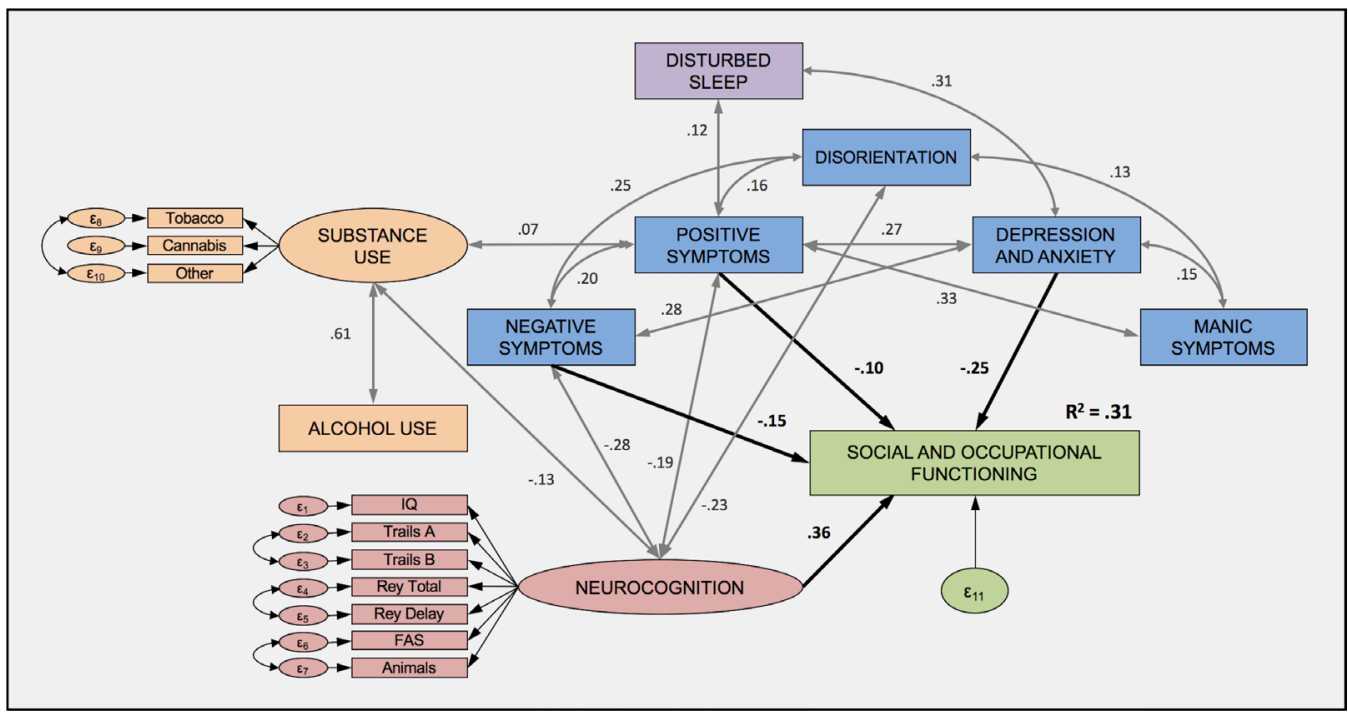

Figure 2 Final model. All unidirectional (correlation) and directional (regression) paths are significant at $p<0.001$ (except correlation between substance use and positive symptoms, where $\mathrm{p}<0.05)$. Where no path is drawn, it denotes no significant relationship between the variables (see figure 1 for all factor loadings of latent variables).

12-year-old to 20-year-old group $(\beta=-0.06, \mathrm{p}=0.178)$. The model with older individuals explained $18 \%$ more variance in functional impairment than the model with younger individuals. This was driven in large part by a difference in predictive strength of neurocognition, whereby it was more predictive in older $(\beta=0.44$, $\mathrm{p}<0.001)$ than younger individuals $(\beta=0.27, \mathrm{p}<0.001)$.

- Gender. Positive symptoms were non-significant in the male subgroup $(\beta=-0.07, p=0.145)$, whereas all other clinical features remained significant ( $p$ values $<0.001)$. In females, negative symptoms became non-significant $(\beta=-0.07, p=0.105)$, while the other contributors remained significant. The final model was comparable across genders in terms of the total variance explained.

- Primary affective disorder diagnosis. Neurocognition, depression and anxiety, and negative symptoms remained significant contributors to functional level irrespective of affective disorder diagnosis (see table 4). By contrast, positive symptoms no longer remained significant in both the affective disorder $(\beta=-0.63, p=0.097)$ and psychosis, developmental or behavioural disorders $(\beta=-0.102, p=0.123)$ subgroups. An additional $14 \%$ of the variance in functioning was explained in individuals with a psychotic, developmental or behavioural disorder, primarily owing to the greater predictive strength of neurocognition ( 0.30 vs $0.43, \mathrm{p}<0.001)$.

- Medication usage. All factors associated with functional impairment remained significant in participants who were unmedicated. By contrast, positive symptoms no longer remained significant in medicated individuals $(\beta=-0.06, p=0.117)$.

\section{Sensitivity analysis}

Restricting the full sample to individuals aged 15-25years $(\mathrm{n}=794)$ yielded a very good fitting model as well $\left(\chi^{2}=240.1\right.$, $\mathrm{d} f=119, \quad \mathrm{p}<0.000$, CFI $=0.959$, NFI $=0.924$, RMSEA $=0.036$, $90 \%$ CI 0.029 to 0.042 ). The explained variance remained the same ( $31 \%$ explained). Importantly, all predictors remained significant with the same effect sizes, with the exception of depression and anxiety, which became slightly more predictive $(-0.25 \rightarrow-0.26)$, and neurocognition, which became slightly less predictive $(0.36 \rightarrow 0.35)$.

Table 3 Analyses of demographic factors (age, gender) as moderators of the relationships between predictors and functional outcome in the final model

\begin{tabular}{|c|c|c|c|c|c|c|c|c|}
\hline & \multicolumn{4}{|l|}{ Age $^{*}$} & \multicolumn{4}{|c|}{ Gender† } \\
\hline & \multicolumn{2}{|c|}{$12-20$ Years $(n=539)$} & \multicolumn{2}{|c|}{$21-36$ Years $(n=464)$} & \multicolumn{2}{|c|}{ Male $(n=461)$} & \multicolumn{2}{|c|}{ Female $(n=542)$} \\
\hline & $\boldsymbol{\beta}$ & $P$ values & $\beta$ & $P$ values & $\boldsymbol{\beta}$ & $P$ values & $\beta$ & $P$ values \\
\hline Neurocognition & 0.27 & 0.000 & 0.44 & 0.000 & 0.35 & 0.000 & 0.35 & 0.000 \\
\hline Depression and anxiety & -0.28 & 0.000 & -0.22 & 0.000 & -0.23 & 0.000 & -0.30 & 0.000 \\
\hline Positive symptoms & -0.06 & 0.178 & -0.14 & 0.002 & -0.07 & 0.145 & -0.12 & 0.004 \\
\hline Negative symptoms & -0.13 & 0.005 & -0.18 & 0.000 & -0.19 & 0.000 & -0.07 & 0.105 \\
\hline
\end{tabular}

*12-20 Years (subgroup Model, $R^{2}=0.24$ ); 21-36 Years (Subgroup Model, $R^{2}=0.40$ ).

†Male (subgroup model, $R^{2}=0.32$ ); female (subgroup model, $R^{2}=0.29$ ). 
Table 4 Analyses of clinical factors (primary affective disorder, medication usage) moderating the relationship between predictors and functional outcome in the final model

\begin{tabular}{|c|c|c|c|c|c|c|c|c|}
\hline & \multicolumn{4}{|c|}{ Primary affective disorder* } & \multicolumn{4}{|c|}{ Medication usage $†$} \\
\hline & \multicolumn{2}{|c|}{ Yes $(n=736)$} & \multicolumn{2}{|c|}{ No $(n=267)$} & \multicolumn{2}{|c|}{ Nil $(n=309)$} & \multicolumn{2}{|c|}{ Medicated $(n=568)$} \\
\hline & $\boldsymbol{\beta}$ & P values & $\boldsymbol{\beta}$ & $P$ values & $\boldsymbol{\beta}$ & P values & $\boldsymbol{\beta}$ & $P$ values \\
\hline Depression and anxiety & -0.29 & 0.000 & -0.24 & 0.000 & -0.15 & 0.007 & -0.24 & 0.000 \\
\hline Positive symptoms & -0.06 & 0.097 & -0.10 & 0.123 & -0.22 & 0.000 & -0.06 & 0.117 \\
\hline
\end{tabular}

*Yes (subgroup model, $R^{2}=0.24$ ); no (subgroup model, $R^{2}=0.38$ ).

†Nil (subgroup model, $R^{2}=0.38$ ); medicated (subgroup model, $R^{2}=0.29$ ).

\section{DISCUSSION}

In a large, clinical, transdiagnostic cohort of youth with mental disorders, impaired neurocognition was the clinical feature most significantly associated with functional impairment. The role of neurocognition was attenuated but still significant in those with an affective disorder diagnosis and in the younger age group. The findings are relevant as they demonstrate that while neurocognitive impairment may undermine functioning in those with psychotic disorders, or in chronic or recurrent mental disorders, they are not specific to such cases. That is, neurocognitive dysfunction has traditionally been argued as a core, underlying feature of social and occupational impairments in chronic schizophrenia. However, our current findings support the burgeoning position that the role of neurocognitive deficits cuts across diagnosis and clinical stage. Nevertheless, it appears that neurocognitive disturbances are more pronounced in those with psychotic, developmental or behavioural disorders, converging with evidence of more pronounced cognitive deficits in children who will go on to develop psychosis compared with those who develop depression or bipolar disorder. ${ }^{49-51}$ Mechanistically, whether neurocognitive dysfunction drives functional impairment as a few past studies have found, ${ }^{728}$ and is conversely a consequence of poor functioning remains to be clarified.

Depressive, anxiety and negative symptom dimensions also contributed significantly to level of social and occupational functioning, supporting previous disorder-specific research. ${ }^{11} 1328$ Importantly, the contributions of these factors to level of functioning were largely independent of one another, and do not appear to be moderated by other clinical or demographic factors. By comparison, the role of positive symptoms diminished considerably in the final model. This finding differs from other research in psychotic and bipolar disorders, and may reflect the lower prevalence of positive symptoms in our cohort in contrast to previous studies. ${ }^{111328}$ However, it was notable that positive symptoms in older, unmedicated females remained significantly associated with functioning. As with neurocognition however, the directionality of findings remains unclear, with some evidence suggesting that it may be bidirectional in the case of negative symptoms. ${ }^{7285}$
Intriguingly, neither alcohol and substance use, nor sleep disturbances, were directly associated with functional impairment, although these factors remained significantly associated with neurocognition and clinical symptoms. Therefore, their role in social and occupational functioning does not appear to be direct, but may operate indirectly (eg, substance use may impair cognition which in turn may impair functioning). The indirect effects of alcohol and substance use, as well as sleep and circadian disruptions, warrant more detailed examination and causal analysis in longitudinal datasets. Moreover, the lack of a direct association between alcohol and substance use and functioning may be related to the domains of neurocognitive functions currently tested. That is, the impact of substance use on functioning may be greatest in other neurocognitive functions that are more directly linked to driving and maintaining alcohol and substance use behaviours, such as those subserved by the fear, reward and self-control circuitries not covered in the current neuropsychological battery (eg, reward-related cue learning, habit formation, response inhibition).

The current findings have important implications for the transdiagnostic, dimensional approach to psychiatry. Research examining the underlying mechanisms of functional impairment in single-diagnosis or dual-diagnosis cohorts have been unable to capture the unique contributions of a comprehensive range of neurocognitive, symptom, sleep and circadian factors, as well as other psychoactive exposures (ie, substance use, prescribed medications). ${ }^{22}$ In particular, neuropsychological studies in psychosis have not routinely and concurrently assessed depression and anxiety symptoms, hypomania and fullthreshold mania, substance misuse and sleep disturbance. That is not to say that categorical, nosological approaches have had little to contribute to the field. Indeed, the key argument underpinning a DSM approach is to allow for comparability across studies and so diagnostic determinations are often necessary. However, in youth, diagnoses tend to be unstable ${ }^{1}$ and, as such, not as useful. One plausible way forward for dimensional psychiatry is to ensure that the samples used in transdiagnostic studies are characterised as clearly and as comprehensively as possible, ${ }^{165354}$ as was attempted in the present investigation. 
In terms of limitations, the current analyses were cross-sectional, and future research investigating moderator and mediator analyses would benefit from crosslagged, longitudinal path modelling to disentangle causality. ${ }^{55}$ Second, the measures used to index clinical symptoms, sleep disturbance, and alcohol and substance use were not as comprehensive as is typical in the sleep and addiction literatures, and some were not originally designed for use in certain clinical disorders which may have reduced sensitivity to detect symptoms (eg, mania). More detailed examination of these dimensions in the future will help more definitively determine whether the impact of neurocognition on functioning is as large as currently identified. Future studies would also benefit from using real-time, ecological momentary assessment technologies (eg, substance use monitoring using smartphones, actigraphy monitoring of physical activity and sleep quality). Third, medication data were not available for the full sample ( $12.6 \%$ were missing) and, as such, the moderating role of medication status requires further corroboration (as with the role of medication type). Fourth, clinical diagnoses assigned to cases in the current study were by treating psychiatrists and future studies should consider more structured approaches (eg, structured clinical interview for DSM), including consideration of the influence of other comorbid diagnoses (eg, personality disorders). Further, the age range included in the current study meant that individuals on the opposite ends of the age spectrum were at different stages of their cognitive and emotional development (eg, executive functioning, emotional regulation), although our sensitivity analyses support the argument that our findings hold irrespective of age. Further, a phenotype-approach, as attempted in the current study, would necessarily require converging genetic and neuroimaging evidence to ensure that the neurocognitive and symptom dimensions identified as predictive of functioning are linked to specific neural circuitries (eg, corticobasal ganglia systems ${ }^{56}$ ) and genotype which would ultimately facilitate the development of next-generation and neuroscience-informed pharmacotherapies. Finally, it remains to be seen whether the current findings hold in future studies with less missing data, as well as in studies using measures or approaches that can circumvent potential biases stemming from non-normally distributed data.

This was the first study to examine a broad range of illness-related factors and associations with functional impairment in a well-powered and broadly transdiagnostic, clinical cohort of more than one thousand young people with mental illness. A significant contribution of the present findings to the established literature was evidence showing that neurocognition is a strong and reliable, unique predictor of social and occupational functioning irrespective of diagnosis-in a cohort predominantly composed of affective disorders which has not been previously demonstrated before at this scale. As such, the functional importance of neurocognitive functions clearly extends beyond the psychosis and developmental disorders spectrums and appears to become more pronounced with increasing age. Future studies should attempt to replicate these findings, as well as to clarify the directions of cause and effect.

\section{Author affiliations}

${ }^{1}$ Brain and Mind Centre, University of Sydney, Sydney, New South Wales, Australia ${ }^{2}$ Brain and Mental Health Research Hub, Monash University, Melbourne, Victoria, Australia

${ }^{3}$ Charles Perkins Centre, University of Sydney, Sydney, New South Wales, Australia

${ }^{4}$ The Monash Alfred Psychiatry Research Centre, Monash University, Melbourne, Victoria, Australia

${ }^{5}$ Marie Bashir Institute, University of Sydney, Sydney, New South Wales, Australia ${ }^{6}$ Academic Psychiatry, Institute of Neuroscience, Newcastle University, Newcastle, UK

${ }^{7}$ Centre for Affective Disorders, Institute of Psychiatry, Psychology and Neuroscience, London, UK

Contributors RSCL, DFH, SLN, AJG, NG, JS, EMS and IBH contributed to the conception of the study. RSCL, DFH, MK undertook the data collection, processing and analysis. RSCL wrote the first draft of the manuscript. All authors contributed to data interpretation, discussion and have approved the final manuscript.

Funding This study was supported by grants from the National Health \& Medical Research Council (NHMRC) including: Centre of Research Excellence (No. 1061043), NHMRC Fellowship (No. 1046899) and Clinical Research Fellowship (No. 402864).

Competing interests None declared.

Patient consent Not required.

Ethics approval This study was approved by the University of Sydney Human Research Ethics Committee (reference 12130; protocol no. 2012/1631).

Provenance and peer review Not commissioned; externally peer reviewed.

Data sharing statement The datasets generated and/or analysed during the current study are not publicly available due to the breadth and sensitivity of data collected, which may have implications for individual privacy, but are available from the corresponding author on reasonable request.

Open access This is an open access article distributed in accordance with the Creative Commons Attribution Non Commercial (CC BY-NC 4.0) license, which permits others to distribute, remix, adapt, build upon this work non-commercially, and license their derivative works on different terms, provided the original work is properly cited, appropriate credit is given, any changes made indicated, and the use is non-commercial. See: http://creativecommons.org/licenses/by-nc/4.0/.

\section{REFERENCES}

1. Bromet EJ, Kotov R, Fochtmann LJ, et al. Diagnostic shifts during the decade following first admission for psychosis. Am J Psychiatry 2011;168:1186-94.

2. Hickie IB, Scott J, Hermens DF, et al. Clinical classification in mental health at the cross-roads: which direction next? BMC Med 2013;11:125.

3. McGorry PD, Hickie IB, Yung AR, et al. Clinical staging of psychiatric disorders: a heuristic framework for choosing earlier, safer and more effective interventions. Aust N Z J Psychiatry 2006;40:616-22.

4. Cross SP, Hermens DF, Scott EM, et al. A clinical staging model for early intervention youth mental health services. Psychiatr Serv 2014;65:939-43.

5. Lee RS, Redoblado-Hodge MA, Naismith SL, et al. Cognitive remediation improves memory and psychosocial functioning in firstepisode psychiatric out-patients. Psychol Med 2013;43:1161-73.

6. Cross SPM, Scott J, Hickie IB. Predicting early transition from subsyndromal presentations to major mental disorders. BJPsych Open 2017;3:223-7.

7. Lee RSC, Hermens DF, Scott J, et al. A transdiagnostic study of education, employment, and training outcomes in young people with mental illness. Psychol Med 2017;47:2061-70.

8. O'Dea B, Lee RS, McGorry PD, et al. A prospective cohort study of depression course, functional disability, and NEET status in help-seeking young adults. Soc Psychiatry Psychiatr Epidemiol 2016;51:1395-404. 
9. Davidson L, O'Connell MJ, Tondora J, et al. Recovery in serious mental illness: a new wine or just a new bottle? Prof Psychol 2005;36:480-7

10. World Health Organization. Mental health action plan 2013-2020. Geneva, Switzerland: World Health Organization, 2013.

11. Bowie CR, Depp C, McGrath JA, et al. Prediction of real-world functional disability in chronic mental disorders: a comparison of schizophrenia and bipolar disorder. Am J Psychiatry 2010;167:1116-24.

12. Lee RS, Hermens DF, Redoblado-Hodge MA, et al. Neuropsychological and socio-occupational functioning in young psychiatric outpatients: a longitudinal investigation. PLoS One 2013;8:e58176.

13. Galderisi S, Rossi A, Rocca P, et al. The influence of illness-related variables, personal resources and context-related factors on reallife functioning of people with schizophrenia. World Psychiatry 2014;13:275-87.

14. Thomas ML, Green MF, Hellemann G, et al. Modeling deficits from early auditory information processing to psychosocial functioning in Schizophrenia. JAMA Psychiatry 2017;74:37-46.

15. Whiteford HA, Degenhardt L, Rehm J, et al. Global burden of disease attributable to mental and substance use disorders: findings from the Global Burden of Disease Study 2010. The Lancet 2013;382:1575-86.

16. Cuthbert BN. The RDoC framework: facilitating transition from ICD/ DSM to dimensional approaches that integrate neuroscience and psychopathology. World Psychiatry 2014;13:28-35.

17. Robbins TW, Gillan CM, Smith DG, et al. Neurocognitive endophenotypes of impulsivity and compulsivity: towards dimensional psychiatry. Trends Cogn Sci 2012;16:81-91.

18. Millan MJ, Agid $\mathrm{Y}$, Brüne $\mathrm{M}, \mathrm{Br} \tilde{\mathrm{A}} 1 / 4$ ne $\mathrm{M}$, et al. Cognitive dysfunction in psychiatric disorders: characteristics, causes and the quest for improved therapy. Nat Rev Drug Discov 2012;11:141-68.

19. Hägele C, Schlagenhauf F, Rapp M, et al. Dimensional psychiatry: reward dysfunction and depressive mood across psychiatric disorders. Psychopharmacology 2015;232:331-41.

20. Yee CM, Javitt DC, Miller GA. Replacing DSM categorical analyses with dimensional analyses in psychiatry research: the research domain criteria initiative. JAMA Psychiatry 2015;72:1159-60.

21. Miller GA. Mistreating psychology in the decades of the brain Perspect Psychol Sci 2010;5:716-43.

22. Iorfino F, Hickie IB, Lee RS, et al. The underlying neurobiology of key functional domains in young people with mood and anxiety disorders: a systematic review. BMC Psychiatry 2016;16:1-38.

23. Lee RS, Hermens DF, Naismith SL, et al. Neuropsychological and functional outcomes in recent-onset major depression, bipolar disorder and schizophrenia-spectrum disorders: a longitudinal cohort study. Transl Psychiatry 2015;5:e555.

24. Hermens DF, Naismith SL, Lagopoulos J, et al. Neuropsychological profile according to the clinical stage of young persons presenting for mental health care. BMC Psychol 2013;1:8.

25. Scott EM, Hermens DF, Glozier N, et al. Targeted primary carebased mental health services for young Australians. Med J Aust 2012;196:136-40.

26. Goldman HH, Skodol AE, Lave TR. Revising axis $V$ for DSMIV: a review of measures of social functioning. Am J Psychiatry 1992;149:1148-56.

27. Lee RS, Hermens DF, Redoblado-Hodge MA, et al Neuropsychological and socio-occupational functioning in young psychiatric outpatients: a longitudinal investigation. PLoS One 2013;8.

28. Green MF, Hellemann G, Horan WP, et al. From perception to functional outcome in schizophrenia: modeling the role of ability and motivation. Arch Gen Psychiatry 2012;69:1216-24.

29. Strauss E, Sherman EMS, Spreen O. A compendium of neuropsychological tests: administration, norms, and commentary. 3rd ed. New York: Oxford University Press, 2006

30. Lezak MD, Howieson DB, Bigler ED, et al; Neuropsychological assessment. 5th ed. New York: Oxford University Press, 2012.

31. Dazzi F, Shafer A, Lauriola M. Meta-analysis of the Brief Psychiatric Rating Scale - Expanded (BPRS-E) structure and arguments for a new version. J Psychiatr Res 2016;81:140-51.
32. Londborg PD, Smith WT, Glaudin V, et al. Short-term cotherapy with clonazepam and fluoxetine: anxiety, sleep disturbance and core symptoms of depression. J Affect Disord 2000;61:73-9.

33. Hamilton M. A rating scale for depression. Journal of Neurology, Neurosurgery \& Psychiatry 1960;23:56-62.

34. Saunders JB, Aasland OG, Babor TF, et al. Development of the Alcohol Use Disorders Identification Test (AUDIT): WHO collaborative project on early detection of persons with harmful alcohol consumption--II. Addiction 1993;88:791-804.

35. WHO ASSIST Working Group. The Alcohol, Smoking and Substance Involvement Screening Test (ASSIST): development, reliability and feasibility. Addiction 2002;97:1183-94.

36. Arbuckle JL. IBM SPSS Amos 20 User's Guide: IBM Corporation, 2011.

37. Miot HA. Avaliação da normalidade dos dados em estudos clínicos e experimentais. J Vasc Bras 2017;16:88-91.

38. Muthén B, Kaplan D. A comparison of some methodologies for the factor analysis of non-normal Likert variables. Br J Math Stat Psychol 1985;38:171-89.

39. Bentler PM. Comparative fit indexes in structural models. Psychol Bull 1990;107:238-46.

40. Bentler PM, Bonett DG. Significance tests and goodness of fit in the analysis of covariance structures. Psychol Bull 1980;88:588-606.

41. Steiger $\mathrm{JH}$. Tests for comparing elements of a correlation matrix. Psychol Bull 1980;87:245-51.

42. Schermelleh-Engel K, Moosbrugger H, Müller H. Evaluating the fit of structural equation models: Tests of significance and descriptive goodness-of-fit measures. Methods of Psychological ResearchOnline 2003;8:23-74.

43. Jöreskog K, Sörbom D. LISREL 8: structural equation modeling with the SIMPLIs command language. Chicago: Scientific Software International Inc, 1993

44. Wheaton B, Muthen B, Alwin DF, et al. Assessing reliability and stability in panel models. Sociol Methodol 1977;8:84-136.

45. Tabachnick B, Fidell L. Using multivariate statistics. 5th ed. New York: Allyn and Bacon, 2007.

46. Kline R. Principles and practice of structural equation modeling. 2nd ed. New York: The Guilford Press, 2005.

47. Lee RS, Hermens DF, Porter MA, et al. A meta-analysis of cognitive deficits in first-episode major depressive disorder. $J$ Affect Disord 2012;140:113-24.

48. Lee RS, Hermens DF, Scott J, et al. A meta-analysis of neuropsychological functioning in first-episode bipolar disorders. $J$ Psychiatr Res 2014;57:1-11.

49. Reichenberg A, Weiser M, Rabinowitz J, et al. A population-based cohort study of premorbid intellectual, language, and behavioral functioning in patients with schizophrenia, schizoaffective disorder, and nonpsychotic bipolar disorder. Am J Psychiatry 2002;159:2027-35

50. Koenen KC, Moffitt TE, Roberts AL, et al. Childhood IQ and adult mental disorders: a test of the cognitive reserve hypothesis. Am J Psychiatry 2009;166:50-7.

51. Zammit S, Allebeck P, David AS, et al. A longitudinal study of premorbid IQ Score and risk of developing schizophrenia, bipolar disorder, severe depression, and other nonaffective psychoses. Arch Gen Psychiatry 2004;61:354-60.

52. Nakagami E, Hoe M, Brekke JS. The prospective relationships among intrinsic motivation, neurocognition, and psychosocial functioning in schizophrenia. Schizophr Bull 2010;36:935-48.

53. Casey BJ, Craddock N, Cuthbert BN, et al. DSM-5 and RDoC: progress in psychiatry research? Nat Rev Neurosci 2013;14:810-4.

54. Craddock N, Owen MJ. Rethinking psychosis: the disadvantages of a dichotomous classification now outweigh the advantages. World Psychiatry 2007;6:84-91.

55. Breitborde NJ, Srihari VH, Pollard JM, et al. Mediators and moderators in early intervention research. Early Interv Psychiatry 2010;4:143-52.

56. Griffiths KR, Lagopoulos J, Hermens DF, et al. Impaired causal awareness and associated cortical-basal ganglia structural changes in youth psychiatric disorders. Neuroimage Clin 2016;12:285-92 